\title{
High time for advancing marijuana research
}

\author{
Marijuana use is expected to increase as its legalization spreads. With more marijuana users, we should prioritize \\ research on this pervasive, but relatively understudied, drug.
}

M arijuana is now legal for recreational or medicinal use in 20 states in the United States and has been decriminalized in many others. A recent Gallup poll ${ }^{1}$ found that more than half of Americans surveyed favored legalizing the use of marijuana. Although the extent to which legalization will expand the pool of marijuana users is unknown, it seems likely that there will be a substantial increase; moreover, escalating use in even a small fraction of the US population would represent a big surge in absolute numbers of users. Marijuana has typically not been considered as high a priority for research as more addictive drugs such as opioids and cocaine. However, as marijuana stands poised to join alcohol and tobacco as a legalized drug, it is critical that we prioritize research to improve our understanding of the medicinal, toxicological, addiction and public health implications of increased marijuana use.

The largest increase in legalized marijuana use has been for medical treatment, based on research suggesting that the cannabis plant contains compounds that may have a wide variety of therapeutic applications. Those with otherwise intractable pain, nausea and glaucoma (among other ailments) are understandably eager to take advantage of this new and increasingly legal treatment. However, marijuana itself is not a medicine; it includes a large number of compounds with unknown effects, the identified components may interact or interfere when delivered in combination, and levels of active compounds may vary from strain to strain. These compounds will have wide-ranging effects, as the endocannabinoid system is pervasive in the brain; marijuana can influence mood and is known to impair memory, cognitive function, reaction time and motor coordination. It is important that we prioritize research to determine what other consequences of cannabis use there might be, particularly for long-term use and in those with ailments serious enough to consider marijuana for medical treatment. This information is critical to help the increasing numbers of people with the opportunity to use marijuana make educated decisions about the consequences of choosing to do so.

One particularly under-appreciated consequence of marijuana use is the risk of addiction. The National Institute of Drug Abuse suggests that approximately $9 \%$ of people who use marijuana may become dependent, and this number may be even higher for those who start using as adolescents. Although this rate is far lower than that reported for heroin (23\%), in raw numbers, marijuana dependence is already a far more pervasive problem than heroin addiction: according to a 2012 report from NIDA, there were nearly ten times as many individuals with marijuana abuse problems as there were individuals with heroin abuse problems. This number will only grow as marijuana becomes more easily accessible and acceptable to use, so it is urgent that we increase efforts to understand what factors influence dependence and develop targeted treatments. Currently, there are a few pharmaceutical treatments for opiate, alcohol and nicotine addiction, but there is no such drug for marijuana addiction.
An important strategy for decreasing some of the negative consequences of marijuana use may be increasing efforts to develop drugs based on specific components of cannabis. By narrowing the number of active compounds administered, it may be possible to target particular aspects of the cannabinoid system and avoid some of the side effects. For example, cannabidiol, a non-psychotropic ingredient of marijuana, has shown some promise as an anti-psychotic treatment for schizophrenia. The development of FDA-approved drugs will be particularly urgent as marijuana legalization becomes widespread, as this will also open up opportunities for manufacturers of over-the-counter remedies. As has happened in the weight-loss drug market, these manufacturers will be eager to sell supplements made of entirely legal over-the-counter ingredients, but which have limited evidence of efficacy or safety, and which will be largely unregulated.

Although it is imperative that we advance the marijuana research agenda, there are currently many obstacles that make it difficult for researchers. A key point of pain, as always, is the need for more financial support. US government funds for research have largely stagnated during the continuing budget crises, so scientists are under pressure to do more with less. Pharmaceutical companies may participate in some drug discovery work, but have shown little interest in addiction research and have no incentive at all to examine the negative consequences of medical marijuana use. Government resources will need to be channeled to addressing these critical research questions in order to make progress.

A major impediment to marijuana research is that the substance is still classified by federal law as a schedule I drug (the same category that contains heroin and LSD), making it very difficult for researchers to work with. Acquiring the permissions necessary to do marijuana research can take years and require an enormous burden of paperwork ${ }^{2}$. Legalization is occurring on a state-by-state basis, but the federal policies that govern researchers have not been updated. In light of mounting evidence that marijuana has potential for medical use, it would make sense to reclassify it to a less restrictive category, a change that would dramatically ease constraints on cannabis research.

As the use of marijuana appears to be headed toward wide acceptance in the United States, it is urgent that we support a comprehensive research program addressing the critical gaps in our understanding. If the harmful aspects of marijuana use outweigh the therapeutic benefits, we need to find out now, not far in the future. If there are components of cannabis with specific therapeutic value, we need to develop drugs that target them. Ultimately, we need data and hard facts in hand to responsibly regulate the use of marijuana and educate consumers about their choices.

1. Swift, A. Gallup <http://www.gallup.com/poll/165539/first-time-americans-favor-
legalizing-marijuana.aspx> (2013). 2. Anonymous. Nat. Neurosci. 8, 971 (2005). 